FIAN-30/99

UCRHEP-E255

14 May 1999

revised 24 August 1999

\title{
Energy dependence of mean multiplicities in gluon and quark jets at the next-to-next-to-next-to-leading order
}

\author{
I.M. Dremin ${ }^{1}$ and J.W. Gary ${ }^{2}$ \\ ${ }^{1}$ Lebedev Physical Institute, Moscow 117924, Russia \\ ${ }^{2}$ Department of Physics, University of California, Riverside CA 92521, \\ USA
}

\begin{abstract}
Analytic predictions for the energy dependence of the mean multiplicities in gluon and quark jets are presented at the next-to-next-tonext-to-leading order (3NLO) of perturbative QCD and are compared to experiment. The $3 \mathrm{NLO}$ correction to the gluon jet multiplicity is found to be small. The corresponding theoretical expression provides a good description of available gluon jet measurements. The 3NLO correction to the quark jet multiplicity is large. The theoretical expression for quark jets also describes the data accurately, however, but not with the same parameter values as are used for gluon jets. It is shown that the well known success of the next-to-leading order (NLO) approximation in describing the energy evolution of quark jet multiplicity can be attributed to the equivalence of the quark and gluon expressions at NLO to within a constant factor, and to almost constant contributions from higher order terms to the gluon jet result.
\end{abstract}

pacs: 12.38.Bx,12.38.-t, 13.65.+i, 14.70.Dj

keywords: Jets, Multiplicity, Perturbative QCD, Gluons 
Particle multiplicity is one of the most basic properties of a jet. The multiplicity of a jet has been the subject of many theoretical and experimental studies [1]. Analytic predictions for the energy dependence of mean multiplicities in gluon and quark jets can be derived from perturbative QCD (pQCD). The total multiplicity of a jet, i.e. the multiplicity in full phase space, is subject to important corrections in pQCD and thus provides a sensitive means to test higher order perturbation theory.1 In the present study, we present a test of higher order perturbative calculations based on the total multiplicity in jets.

The perturbative solutions for multiplicity are usually expressed in terms of the anomalous dimension $\gamma$ and the multiplicity ratio $r$, defined by

$$
\gamma(y) \equiv \frac{n_{\mathrm{G}}^{\prime}(y)}{n_{\mathrm{G}}(y)}=\left[\ln n_{\mathrm{G}}(y)\right]^{\prime} \quad ; \quad r(y) \equiv \frac{n_{\mathrm{G}}(y)}{n_{\mathrm{F}}(y)},
$$

where $n_{\mathrm{G}}$ and $n_{\mathrm{F}}$ are the mean total multiplicities in gluon and quark jets, respectively. In these expressions, $y=\ln \left(p \theta / \mathrm{Q}_{0}\right)$ defines the energy scale of the jets, with $p$ the momentum of the parton which initiates the jet, $\theta$ the opening angle of the first branching in the jet, and $\mathrm{Q}_{0}$ a cutoff which defines the limit of perturbative evolution. For $p$ large and $\theta$ small, the virtuality $\mathrm{Q}_{\text {jet }}$ (invariant mass) of the jet is given by $\mathrm{Q}_{\text {jet }} \approx p \theta / 2$. Primed quantities in (四) denote derivatives with respect to $y: n_{\mathrm{G}}^{\prime}=\mathrm{d} n_{\mathrm{G}} / \mathrm{d} y$, etc.

Perturbative expressions for $n_{\mathrm{G}}$ and $n_{\mathrm{F}}$ at the next-to-leading order (NLO) and the next-to-next-to-leading order (NNLO) approximations of pQCD have been presented in [4] and [5], respectively. Here we present the solutions at the next-to-next-to-next-to-leading order (3NLO) approximation and compare the results to experiment.

The perturbative expressions for $\gamma$ and $r$ at 3NLO are:

$$
\begin{aligned}
& \gamma(y)=\gamma_{0}\left(1-a_{1} \gamma_{0}-a_{2} \gamma_{0}^{2}-a_{3} \gamma_{0}^{3}\right)+\mathcal{O}\left(\gamma_{0}^{5}\right) \\
& r(y)=r_{0}\left(1-r_{1} \gamma_{0}-r_{2} \gamma_{0}^{2}-r_{3} \gamma_{0}^{3}\right)+\mathcal{O}\left(\gamma_{0}^{4}\right)
\end{aligned}
$$

with

$$
\gamma_{0}=\sqrt{\frac{2 \alpha_{S} \mathrm{~N}_{\mathrm{C}}}{\pi}}=\sqrt{\frac{4 \mathrm{~N}_{\mathrm{C}}}{\beta_{0} y}\left(1-\frac{\beta_{1} \ln (2 y)}{\beta_{0}^{2} y}\right)},
$$

${ }^{1}$ This is in contrast to multiplicity in a limited region of phase space, such as the multiplicity of soft particles, which is less sensitive to higher order corrections; this fact has recently been exploited to determine the color factor ratio $\mathrm{C}_{\mathrm{A}} / \mathrm{C}_{\mathrm{F}}$ using the soft particle multiplicities in gluon and quark jets [2] (see also [3]). 
where $\alpha_{S}$ is the strong coupling strength, $\beta_{0}=\left(11 \mathrm{~N}_{\mathrm{C}}-2 \mathrm{~N}_{\mathrm{F}}\right) / 3, \beta_{1}=\left(51 \mathrm{~N}_{\mathrm{C}}-\right.$ $\left.19 \mathrm{~N}_{\mathrm{F}}\right) / 3, \mathrm{~N}_{\mathrm{C}}=3$ is the number of colors, $\mathrm{N}_{\mathrm{F}}$ is the number of active quark flavors, $r_{0}=\mathrm{N}_{\mathrm{C}} / \mathrm{C}_{\mathrm{F}}$, and $\mathrm{C}_{\mathrm{F}}=4 / 3$. The coefficients $a_{i}$ and $r_{i}$ are calculable in pQCD and are given in [6] for $i=1,2,3$. []

The expressions for $\gamma(y)$ in (11) and (\#) can be combined to yield

$$
n_{\mathrm{G}}(y) \propto \exp \left[\int^{y}\left(\gamma_{0}-a_{1} \gamma_{0}^{2}-a_{2} \gamma_{0}^{3}-a_{3} \gamma_{0}^{4}\right) \mathrm{d} y\right] \quad .
$$

In conjunction with (4), we then determine the energy dependence of the gluon jet multiplicity at $3 \mathrm{NLO}$ to be:

$$
n_{\mathrm{G}}(y)=\mathrm{K} y^{-a_{1} \mathrm{C}^{2}} \exp \left[2 \mathrm{C} \sqrt{y}+\delta_{\mathrm{G}}(y)\right],
$$

where $\mathrm{K}$ is an overall normalization constant, $\mathrm{C}=\sqrt{4 \mathrm{~N}_{\mathrm{C}} / \beta_{0}}$, and

$$
\begin{aligned}
\delta_{\mathrm{G}}(y)= & \frac{\mathrm{C}}{\sqrt{y}}\left\{2 a_{2} \mathrm{C}^{2}+\frac{\beta_{1}}{\beta_{0}^{2}}[\ln (2 y)+2]\right\} \\
& +\frac{\mathrm{C}^{2}}{y}\left\{a_{3} \mathrm{C}^{2}-\frac{a_{1} \beta_{1}}{\beta_{0}^{2}}[\ln (2 y)+1]\right\} .
\end{aligned}
$$

To obtain this result, the term proportional to $\beta_{1}$ in parenthesis in equation (仼) is treated as being small compared to 1 . The first term in the exponent in equation (6) is the leading order (LO) term. The term in front of the exponential arises at NLO. The term proportional to $1 / \sqrt{y}$ in (7) enters at NNLO, while that proportional to $1 / y$ is the 3 NLO contribution.

Experimental measurements of the inclusive charged particle multiplicity of gluon jets as a function of energy scale Q are shown in Fig. 1. These results utilize a definition of gluon jets which corresponds to that employed for the calculation, based on the production of a virtual gluon jet pair, gg, from a color singlet point source. These are the only such gluon jet data currently available. The three data points at scale $\mathrm{Q} \approx 5 \mathrm{GeV}$ are derived from the hadronic component of $\Upsilon(1 \mathrm{~S}) \rightarrow \gamma$ gg decays $[7]$. The virtuality $\mathrm{Q}$ is given by the invariant mass of the hadronic system. Similarly, $\Upsilon(3 S) \rightarrow \gamma \chi_{b 2}(10.27) \rightarrow$ $\gamma$ gg decays provide the measurement at $\mathrm{Q} \approx 10 \mathrm{GeV}$ [8], with the scale given by the $\chi_{b 2}$ mass. The result at $\mathrm{Q} \approx 80 \mathrm{GeV}$ [2, 9] is based on hadronic $\mathrm{Z}^{0}$ data:

\footnotetext{
${ }^{2}$ For $\mathrm{N}_{\mathrm{F}}=3$, the value we emphasize in this letter, $a_{1}, a_{2}$ and $a_{3}$ are $0.280,-0.379$ and 0.209 , while $r_{1}, r_{2}$ and $r_{3}$ are $0.185,0.426$ and 0.189 [6].
} 
$\mathrm{Z}^{0} \rightarrow \mathrm{q} \overline{\mathrm{q}} \mathrm{g}_{\text {incl. }}$, in which $\mathrm{g}_{\text {incl. }}$ refers to a gluon jet hemisphere recoiling against two almost collinear quark jets $q$ and $\bar{q}$ in the opposite hemisphere. The $g_{\text {incl. }}$ jets correspond closely to hemispheres of color singlet gg events, as discussed in [10]. For the results shown here, the $g_{\text {incl. }}$ hemisphere results reported in [2] have been multiplied by a factor of two both for the multiplicity and energy scale so that they correspond to full gg events analogous to the $\Upsilon$ data.

The solid curve in Fig. 1 shows the result of a $\chi^{2}$ fit of expression (6) to the data, with $y=\ln (\mathrm{Q} / \Lambda)$ where $\Lambda$ is a fitted parameter. The other fitted parameter is the normalization constant $\mathrm{K}$ in (6). For this fit, $\mathrm{N}_{\mathrm{F}}=3$. The $\Upsilon(1 \mathrm{~S})$ measurements [7] are not included in this fit because a systematic uncertainty was not provided for them. Nonetheless, these points are seen to lie near the fitted curve. The results for the fitted parameters are $\Lambda=1.03 \pm 0.24 \mathrm{GeV}$ and $\mathrm{K}=0.288 \pm 0.037$. The uncertainties are defined by the maximum deviations observed when the gluon jet measurements are varied by their one standard deviation uncertainties. These results, along with those found using $\mathrm{N}_{\mathrm{F}}=4$ and 5 , are summarized in the top portion of Table $\mathbb{1}$. The results of the fits with $\mathrm{N}_{\mathrm{F}}=4$ and 5 are virtually indistinguishable from those shown by the solid curve in Fig. 11. Note that the result using $N_{F}=3$ probably has the most physical relevance since $b$ and c quarks are rarely produced in the perturbative evolution of the jets, even for the $\mathrm{Z}^{0}$ data. The dashed curve in Fig. 1 1 shows the prediction of the Herwig parton shower multihadronic event generator [11], version 5.9, for the inclusive charged particle multiplicity of gg events as a function of the center-of-mass energy, $Q=E_{\text {c.m. }}$. The Monte Carlo parameters are the same as those used in [2]. Herwig is seen to describe the data well and to be similar to the 3NLO result.

The analytic expression for quark jet multiplicity can be obtained from (1) and (3):

$$
n_{\mathrm{F}}(y)=\frac{n_{\mathrm{G}}(y)}{r(y)}=\frac{n_{\mathrm{G}}(y)}{r_{0}\left(1-r_{1} \gamma_{0}-r_{2} \gamma_{0}^{2}-r_{3} \gamma_{0}^{3}\right)},
$$

with $n_{\mathrm{G}}(y)$ given by (6). The expression for $r(y)$ in the denominator of (8) is known to give a relatively poor description of data, however. For example, the $3 \mathrm{NLO}$ prediction at the scale of the $\mathrm{Z}^{0}$ is $r \approx 1.7$ [6], about $13 \%$ larger than the experimental result of $1.51 \pm 0.04$ [2]. Therefore, it can be anticipated that the description of quark jet multiplicity provided by (8) will be deficient if the values of $\Lambda$ and $K$ found using the gluon jet data are employed. It should be noted that this problem with $r$ is even worse at lower orders, e.g. the 
NLO and NNLO predictions for $r$ are about 2.1 and 1.8, respectively, in even greater disagreement with experiment than the 3 NLO result.

Experimental measurements of quark jet multiplicity are shown in Fig. 2 [12]. These data are the inclusive charged particle multiplicity values of $\mathrm{e}^{+} \mathrm{e}^{-} \rightarrow\left(\mathrm{Z}^{0} / \gamma\right)^{*} \rightarrow$ hadrons events and correspond to the definition of quark jets employed for the calculation, i.e. the production of a virtual $q \bar{q}$ pair from a color singlet. The scale is $\mathrm{Q}=\mathrm{E}_{\text {c.m. }}$. The results shown for the LEP experiments are combined values of ALEPH, DELPHI, L3 and OPAL. The combined values are obtained using the unconstrained averaging procedure described in [13], for which a common systematic uncertainty is defined by the unweighted mean of the systematic uncertainties quoted by the experiments. LEP-1 refers to data collected at the $\mathrm{Z}^{0}$ peak, LEP-1.5 to data collected at $\mathrm{E}_{\text {c.m. }} \approx 133 \mathrm{GeV}$, and LEP-2 to data collected at or above the threshold for $\mathrm{W}^{+} \mathrm{W}^{-}$production.

The vertically striped band in Fig. 2 shows the prediction of (8) for $\mathrm{N}_{\mathrm{F}}=3$ using the values of $\Lambda$ and $K$ from the fit to the gluon jet measurements (Fig. 1). The width of the band corresponds to the uncertainties in the values of $\Lambda$ and $\mathrm{K}$ presented above. Almost identical results to those shown by the band are obtained using $\mathrm{N}_{\mathrm{F}}=4$ or 5 . The band typically lies $15-20 \%$ below the data mostly as a consequence of the problem with the theoretical prediction for $r$ noted above.

It is also of interest to fit expression (8) to the quark jet measurements. The results of such a fit using $\mathrm{N}_{\mathrm{F}}=3$ are shown by the solid curve in Fig. 2 . The values obtained for $\Lambda$ and $\mathrm{K}$ are $0.35 \pm 0.12 \mathrm{GeV}$ and $0.222 \pm 0.030$, respectively, where the uncertainties are defined by the maximum difference between the results of the standard fit and those found by fitting only data between 29 and $189 \mathrm{GeV}$, between 12 and $161 \mathrm{GeV}$, or by excluding the LEP-1 data point. The fit is seen to yield a good description of the data. The results found using $\mathrm{N}_{\mathrm{F}}=4$ or 5 are essentially identical to those shown by the solid curve in Fig. 2. The values of the fitted parameters are summarized in the central portion of Table 11. The values of $\Lambda$ obtained from fitting the quark jet data are seen to be three to four times smaller than those obtained from fitting the gluon jet data.

The dashed curve in Fig. 2 shows the prediction of Herwig for the inclusive charged particle multiplicity of hadronic events in $\mathrm{e}^{+} \mathrm{e}^{-}$annihilations. The Herwig prediction reproduces the measurements well, analogously to what was observed for gluon jets.

The expression for $n_{\mathrm{F}}$ given by equation (8) is not entirely satisfactory 
from a theoretical perspective. The purely perturbative $3 \mathrm{NLO}$ result for $n_{\mathrm{F}}$, analogous to equation (6) for $n_{\mathrm{G}}$, is

$$
n_{\mathrm{F}}(y)=\frac{\mathrm{K}}{r_{0}} y^{-a_{1} \mathrm{C}^{2}} \exp \left[2 \mathrm{C} \sqrt{y}+\delta_{\mathrm{F}}(y)\right],
$$

with

$$
\begin{aligned}
\delta_{\mathrm{F}}(y)= & \frac{\mathrm{C}}{\sqrt{y}}\left\{r_{1}+2 a_{2} \mathrm{C}^{2}+\frac{\beta_{1}}{\beta_{0}^{2}}[\ln (2 y)+2]\right\} \\
& +\frac{\mathrm{C}^{2}}{y}\left\{a_{3} \mathrm{C}^{2}+\frac{r_{1}^{2}}{2}+r_{2}-\frac{a_{1} \beta_{1}}{\beta_{0}^{2}}[\ln (2 y)+1]\right\} .
\end{aligned}
$$

where the same notation is used as in equations (6) and (7). Equation (9) is derived from equation (\$), keeping terms only up to $y^{-1}$ in the exponent. It is seen that a term proportional to $r_{3}$ appears in (8) but not in (9): such a term would be proportional to $y^{-3 / 2}$ in (10) and thus would contribute to the 4 NLO approximation. This difference between (8) and (9) arises because of the difference in the order of $\gamma_{0}$ in (2) and (3). The manner in which the powers of $\gamma_{0}$ are structured in (2) and (3) is conventional because $\gamma$ enters in the exponent whereas $r$ is used as a multiplicative factor. As a consequence of this difference between (2) and (3), all corrections of a given order in the denominator of (8) contribute to yet higher orders in (9). For example, $r_{1}$ contributes only to the NLO term in the denominator of (8) whereas it contributes to the NNLO $\left(\sim y^{-1 / 2}\right)$ and 3NLO $\left(\sim y^{-1}\right)$ terms in (9). This situation can also be considered to arise from the fact that the logarithmic slope of quark jets, $\gamma_{\mathrm{F}}$, is given by

$$
\gamma_{\mathrm{F}} \equiv\left[\ln n_{\mathrm{F}}\right]^{\prime}=\gamma-\frac{r^{\prime}}{r}
$$

However, from (3) and (4), $r^{\prime} \approx-r_{0} r_{1} \gamma_{0}^{\prime} \sim r_{0} r_{1} \gamma_{0}^{3}$. Therefore, the first correction to $\gamma_{\mathrm{F}}$ appears at NNLO, but it is determined by the NLO correction to $r$. We conclude that it is improper to use the term with $r_{3}$ in (8) until the $4 \mathrm{NLO}$ contribution to $\gamma$ is known. By extension this implies that if the NLO formula is used to describe quark jet multiplicity, as is common practice (see below), the LO result $r=r_{0}$ should be inserted into all formulas to be self-consistent within the perturbative approach. Therefore the mean multiplicities in quark and gluon jets coincide in NLO up to the constant 
normalization factor $r_{0}=9 / 4$. Any usage of $r$ different from $r_{0}$ implies that NNLO corrections have been included.

The dotted curve in Fig. 20 shows the result of a $\chi^{2}$ fit of equation (9) to the quark jet data for $\mathrm{N}_{\mathrm{F}}=3$. The latter equation is seen to provide a good description of the measurements, equivalent to that given by equation (8). The fitted curves for $\mathrm{N}_{\mathrm{F}}=4$ and 5 are indistinguishable from the curve for $\mathrm{N}_{\mathrm{F}}=3$. The values of the fitted parameters are given in the bottom section of Table 11. These values are similar to those found from fitting equation (8) to the quark jet data.

Our method of comparing the $3 \mathrm{NLO}$ predictions to data is in contrast to the procedure usually employed to test QCD analytic predictions for multiplicity. Typically, the NLO expression for gluon jets [4] is used to fit quark jet data. A fit to gluon jet data is rarely performed. At NLO, the theoretical expressions for $n_{\mathrm{F}}$ and $n_{\mathrm{G}}$ differ by only the constant factor $r_{0}$, as noted above, which justifies this procedure. The NLO expression is found to provide a good description of the measurements, analogous to the agreement we find in the fit of equations (8) or (9) to quark jets (the solid or dotted curves in Fig. 2). This good description belies the fact that the appropriate prediction for $r$ at this order, $r_{0}=2.25$, is in striking disagreement with the experimental value $r \approx 1.5$.

The good description of quark jet multiplicity at NLO is easily understood, because the sum of the NNLO and $3 \mathrm{NLO}$ corrections to $n_{\mathrm{G}}$, given by $\delta_{\mathrm{G}}(y)$ (equation (0) ), is small and almost constant at presently accessible energies. To illustrate this, we show in Fig. 3 the behavior of $\delta_{\mathrm{G}}(y)$ as a function of scale for $\mathrm{N}_{\mathrm{F}}=3,4$ and 5 . For the results of this figure, the scale is $y=\ln (\mathrm{Q} / \Lambda)$ for which the values of $\Lambda$ are taken from the fits to quark jet data using equation (8) (central section of Table 11) to correspond to the procedure outlined above to test the NLO equation. It is seen that $\delta_{\mathrm{G}}$ is almost constant for the range of energies relevant to experiments. Furthermore, $\delta_{\mathrm{G}}$ is small compared to the LO term in the exponent of (6), which typically has a value from 5 to 7 over the range of scale Q shown. Therefore the NLO formula for $n_{\mathrm{G}}$ is not modified significantly by the NNLO and $3 \mathrm{NLO}$ contributions and the expression $n_{\mathrm{F}}=n_{\mathrm{G}} / r$ fits the quark jet data well irrespective of whether the higher order terms are included in $n_{\mathrm{G}}$.

In summary, we have presented analytic expressions for gluon and quark jet multiplicity as a function of energy scale at the next-to-next-to-next-toleading order (3NLO) of perturbation theory. We have performed fits of these expressions to gluon and quark jet measurements, using data for which the 
experimental and theoretical definitions of jets coincide. To our knowledge, this is the first time such a fit has been performed for gluon jets. We find that the gluon jet expression describes the available gluon jet measurements accurately, with values of the parameter $\Lambda$ in the range from about 0.6 to $1.0 \mathrm{GeV}$ depending on the number of active quark flavors, $\mathrm{N}_{\mathrm{F}}$. The $3 \mathrm{NLO}$ expression for quark jets also provides an accurate description of the data with values of $\Lambda$ about three times smaller than those found for gluon jets. We attribute the well known agreement of the next-to-leading order (NLO) expression for quark jet multiplicity with data to the facts that the gluon and quark jet predictions are equivalent at this order to within the constant factor $r_{0}=9 / 4$, and that the gluon jet prediction is only slightly modified by the NNLO and 3NLO terms. 


\section{References}

[1] See, for example, I.M. Dremin, Physics-Uspekhi 37 (1994) 715;

V.A. Khoze and W. Ochs, Int. J. Mod. Phys. A12 (1997) 2949, and references therein.

[2] OPAL Collaboration, G. Abbiendi et al., CERN-EP/99-028, in press in Eur. Phys. J. C.

[3] DELPHI Collaboration, P. Abreu et al., Phys. Lett. B449 (1999) 383.

[4] B.R. Webber, Phys. Lett. B143 (1984) 501.

[5] I.M. Dremin and V.A. Nechitailo, Mod. Phys. Lett. A9 (1994) 1471.

[6] A Capella, I.M. Dremin, J.W. Gary, V.A. Nechitailo, J. Tran Thanh Van, submitted to Nucl. Phys. B.

[7] CLEO Collaboration, M.S. Alam et al., Phys. Rev. D56 (1997) 17.

[8] CLEO Collaboration, M.S. Alam et al., Phys. Rev. D46 (1992) 4822.

[9] OPAL Collaboration, G. Alexander et al., Phys. Lett. B388 (1996) 659;

OPAL Collaboration, K. Ackerstaff et al., Eur. Phys. J. C1 (1998) 479.

[10] J.W. Gary, Phys. Rev. D49 4503 (1994).

[11] G. Marchesini, B.R. Webber et al., Comp. Phys. Comm. 67465 (1992).

[12] JADE Collaboration, W. Bartel et al., Z. Phys. C20 (1983) 187;

TASSO Collaboration, W. Braunschweig et al., Z. Phys. C45 (1989) 193;

HRS Collaboration, M. Derrick et al., Phys. Rev. D34 (1986) 3304;

AMY Collaboration, H.W. Zheng et al., Phys. Rev. D42 (1990) 737;

ALEPH note 98-014 (contribution to the summer conferences);

ALEPH LEPC presentation, November 1998;

DELPHI Collaboration, P. Abreu et al., Eur. Phys. J. C6 (1999) 19;

DELPHI Collaboration, P. Abreu et al., Phys. Lett. B372 (1996) 172;

DELPHI Collaboration, P. Abreu et al., Phys. Lett. B416 (1998) 233;

L3 Collaboration, B. Adeva et al., Z. Phys. C55 (1992) 39;

L3 Collaboration, M. Acciarri et al., Phys. Lett. B371 (1996) 137;

L3 Collaboration, M. Acciarri et al., Phys. Lett. B404 (1997) 390; 
L3 Collaboration, M. Acciarri et al., Phys. Lett. B444 (1998) 569;

OPAL Collaboration, K. Ackerstaff et al., CERN-EP/98-089;

OPAL Collaboration, G. Alexander et al., Z. Phys. C72 (1996) 191;

OPAL Collaboration, K. Ackerstaff et al., Z. Phys. C75 (1997) 193;

OPAL Physics Note PN281 (Feb. 1997);

OPAL Physics Note PN323 (Nov. 1997);

OPAL LEPC presentation, November 1998.

[13] Review of Particle Physics, Eur. Phys. J. C3 (1998) 1. 


\begin{tabular}{|l|cll|}
\hline & $\Lambda(\mathrm{GeV})$ & \multicolumn{1}{c|}{$\mathrm{K}$} & $\chi^{2}$ d.o.f. \\
\hline \hline \multicolumn{4}{|c|}{ (a) Gluon jets, equation (6) } \\
\hline $\mathrm{N}_{\mathrm{F}}=3$ & $1.03 \pm 0.24$ & $0.288 \pm 0.037$ & $0.01 / 2$ \\
$\mathrm{~N}_{\mathrm{F}}=4$ & $0.84 \pm 0.21$ & $0.244 \pm 0.034$ & $0.01 / 2$ \\
$\mathrm{~N}_{\mathrm{F}}=5$ & $0.64 \pm 0.17$ & $0.205 \pm 0.031$ & $0.01 / 2$ \\
\hline (b) Quark jets, equation (8) & \\
\hline $\mathrm{N}_{\mathrm{F}}=3$ & $0.35 \pm 0.12$ & $0.222 \pm 0.030$ & $5.8 / 13$ \\
$\mathrm{~N}_{\mathrm{F}}=4$ & $0.233 \pm 0.086$ & $0.170 \pm 0.024$ & $5.9 / 13$ \\
$\mathrm{~N}_{\mathrm{F}}=5$ & $0.135 \pm 0.055$ & $0.126 \pm 0.019$ & $6.1 / 13$ \\
\hline (b) Quark jets, equation (9) & \\
\hline $\mathrm{N}_{\mathrm{F}}=3$ & $0.322 \pm 0.098$ & $0.216 \pm 0.025$ & $5.6 / 13$ \\
$\mathrm{~N}_{\mathrm{F}}=4$ & $0.231 \pm 0.083$ & $0.169 \pm 0.024$ & $5.8 / 13$ \\
$\mathrm{~N}_{\mathrm{F}}=5$ & $0.148 \pm 0.059$ & $0.129 \pm 0.020$ & $6.1 / 13$ \\
\hline
\end{tabular}

Table 1: Results of a two parameter fit of QCD expressions for the scale evolution of event multiplicity to the measured mean charged particle multiplicities of (a) gg (top), and (b) and (c) q $\bar{q}$ events (center and bottom) from a color singlet point source. For the gluon jets, the QCD expression used is the exact $3 \mathrm{NLO}$ result for $n_{\mathrm{G}}$, equation (6). For the quark jets, the QCD expression is given either by (b) $n_{\mathrm{F}}=n_{\mathrm{G}} / r$, equation (8), which contains some terms beyond $3 \mathrm{NLO}$ as discussed in the text, or by (c) the exact $3 \mathrm{NLO}$ result for $n_{\mathrm{F}}$, equation (9). $\Lambda$ is an energy scale parameter related to the perturbative cutoff $\mathrm{Q}_{0}$ while $\mathrm{K}$ is an overall normalization constant. For the gluon jets, the uncertainties of $\Lambda$ and $\mathrm{K}$ are evaluated by varying the gluon jet data points by \pm 1 standard deviation of their total uncertainties. For the quark jets, the uncertainties are evaluated by varying the fit range as described in the text. 


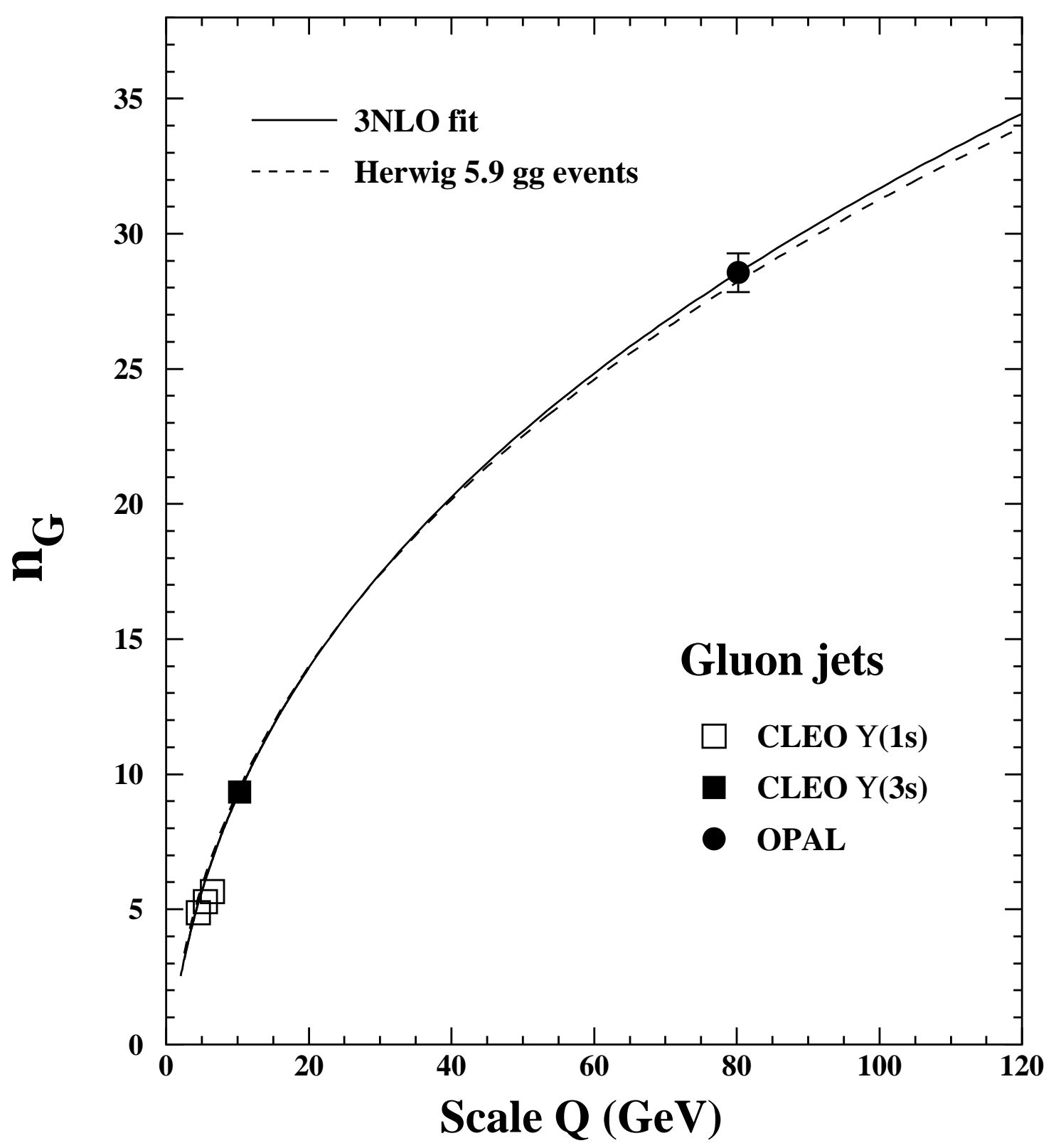

Figure 1: The mean charged particle multiplicity of gg events from a color singlet point source versus energy scale Q. The solid curve shows a fit of the 3 NLO expression to the data using $N_{F}=3$, where $N_{F}$ is the number of active quark flavors. The dashed curve shows the prediction of the Herwig Monte Carlo for gg events. 


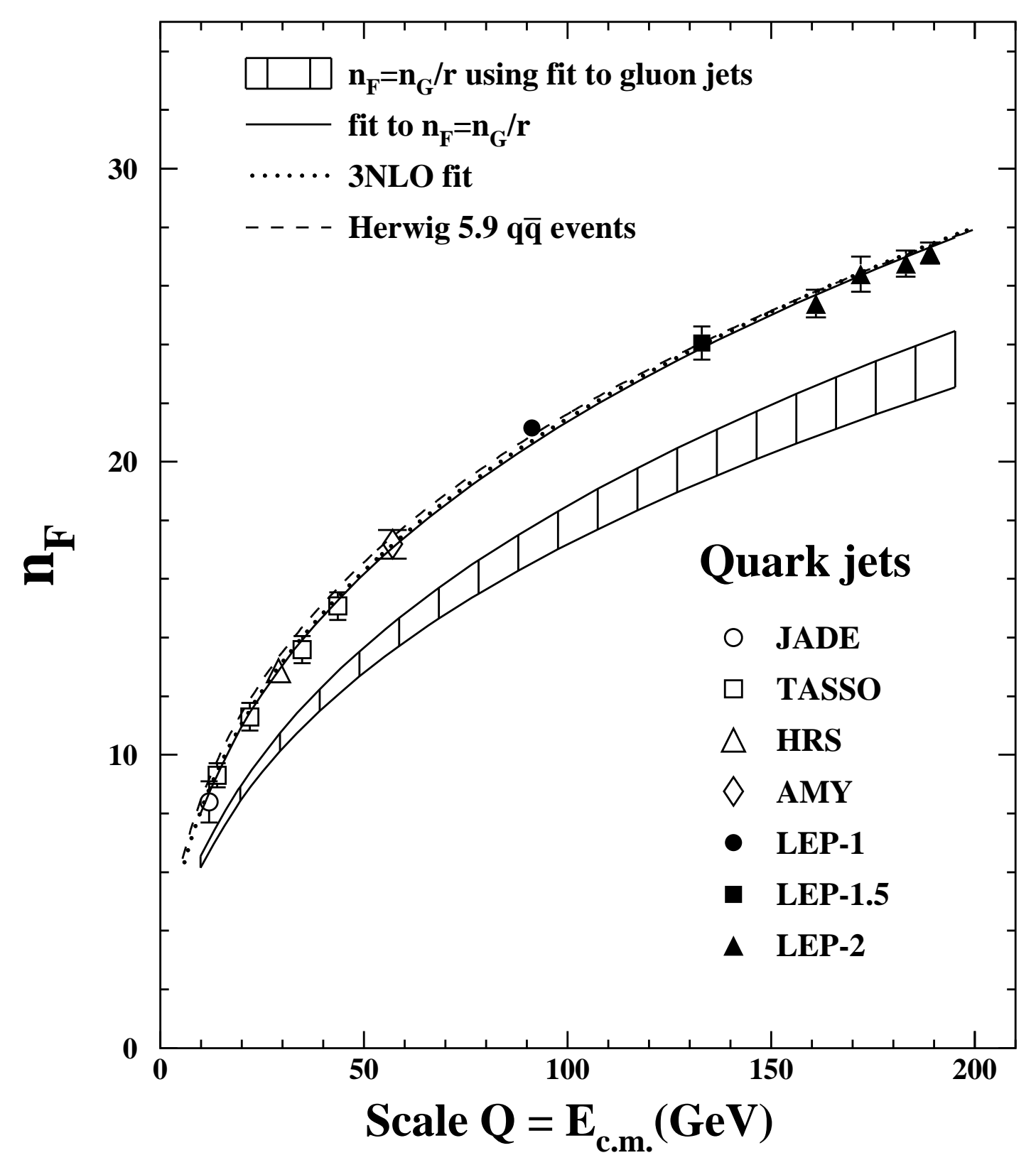

Figure 2: The mean charged particle multiplicity of $\mathrm{e}^{+} \mathrm{e}^{-}$hadronic annihilation events versus energy scale $\mathrm{Q}=\mathrm{E}_{\mathrm{c} . \mathrm{m}}$. The dotted curve shows a fit of the 3NLO expression to the data using $\mathrm{N}_{\mathrm{F}}=3$. The hatched band and solid curve show results using the expression $n_{\mathrm{F}}=n_{\mathrm{G}} / r$ as explained in the text. The dashed curve shows the prediction of the Herwig Monte Carlo for $\mathrm{e}^{+} \mathrm{e}^{-} \rightarrow \mathrm{q} \overline{\mathrm{q}}$ events. 


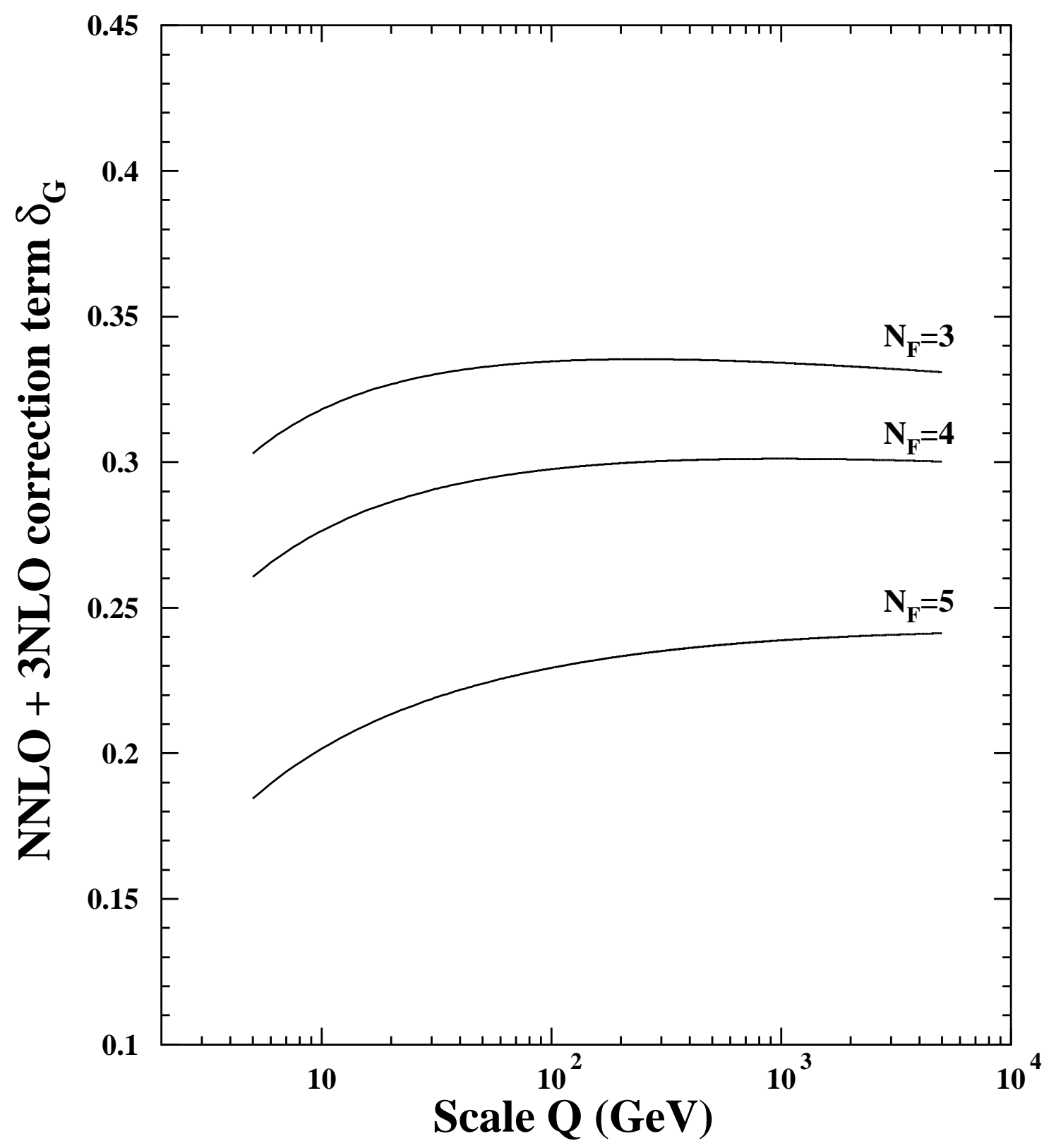

Figure 3: The behavior of the sum of the NNLO and 3NLO correction term to the gluon jet multiplicity, $\delta_{\mathrm{G}}$, as a function of energy scale $\mathrm{Q}$, for $\mathrm{N}_{\mathrm{F}}=3$, 4 and 5. 